\title{
Moment Based Analysis of Equal Gain Combiner in Equally Correlated Nakagami-m Fading Channels
}

\author{
Yunxia Chen \\ Department of Electrical and Computer Engineering \\ University of California, Davis, CA \\ 95616, USA \\ Email: yxchen@ece.ucdavis.edu
}

\author{
Chintha Tellambura \\ Department of Electrical and Computer Engineering \\ University of Alberta, Edmonton, AB, Canada T6G 2V4 \\ Ph:1 7804927228 Fax:1 7804921811 \\ Email: chintha@ece.ualberta.ca
}

\begin{abstract}
Moments of the equal gain combiner (EGC) output signal-to-noise ratio (SNR) are only known for independent and exponentially correlated Nakagami- $m$ fading channels. In this paper, we derive the moments of the EGC output SNR in equally correlated Nakagami- $m$ fading channels. Our moment expressions can be used to evaluate the outage and the average error rate of EGC as well as purely moments-based measures such as the average output SNR and the amount of fading as functions of the fading correlation. Numerical results that illustrate the effect of fading correlation on the distribution of the EGC output SNR are also provided. The average output SNR increases with the fading correlation.
\end{abstract}

Keywords: Amount of fading, diversity, equal gain combining, equally correlated fading.

\section{INTRODUCTION}

The coherent EGC scheme offers performance comparable to the optimal maximal ratio combining (MRC) scheme but with greater simplicity [1]. However, it is notoriously difficult to analyze the EGC performance. Even for independent fading, a closed-form solution to the probability density function (pdf) of the EGC output SNR is only known for the dual-branch case in Rayleigh fading [2]. Different methods have therefore been developed to analyze the EGC performance [2]-[5]. While progress has been made on analyzing the EGC performance in independent fading channels, little is known about that in correlated fading channels.

However, in real-life applications, fading is correlated among diversity branches and hence characterizing the resultant EGC performance loss is of both theoretical and practical importance. Since the joint pdf of the multiple correlated channel gains is not known, many published results deal with the dual-branch case [6]-[9]. For instance, Tellambura and Annamalai [7], [8] derive the average error rate via the characteristic function (chf) of the EGC output SNR. Iskander and Mathiopoulos [9] derive an infinite series expression for the symbol error rate (SER) of QAM in terms of the Appell hypergeometric functions. Accordingly, only several papers address multi-branch $(L>2)$ coherent EGC in correlated fading channels. Karagiannidis et. al. [10] derive the moments of the EGC output SNR in exponentially correlated Nakagami- $m$ fading channels using the available joint pdf of the multivariate Rayleigh distribution. More recently, Chen and Tellambura [11], [12] developed a new approach for performance analysis of multi-branch diversity combiners in equally correlated Rayleigh and Ricean fading channels. They transform a set of equally correlated Rayleigh and Ricean RVs into a set of conditionally independent Ricean RVs using novel representations of the channel gains.

Clearly, it is difficult to derive analytical expressions for the pdf or the mgf of the EGC output SNR in correlated fading channels. In this paper, we thus derive the moments of the EGC output SNR in equally correlated Nakagami- $m$ fading channels using a novel channel gain representation. The equally correlated model presents a worst case among correlated fading channels. It can also be used to describe the correlation among closely-placed antennas [13]. The EGC output moments are important for several reasons. First, purely moment-based measures such as the average output SNR and the amount of fading are commonly used to characterize diversity systems; for example, higher-order moments are derived for certain diversity systems [14]. Second, more widely used performance measures such as the average bit error rate (BER) (which is appropriate for digital modulations) and the outage probability are typically computed using the pdf or the mgf (which are not available in this case). However, these measures can also be evaluated directly via the moments, using the Edgeworth series and Gaussian quadrature techniques.

This paper is organized as follows. Section 2 describes the system and the channel models and develops new representations for the equally correlated Nakagami- $m$ envelopes. Section 3 derives the moments of the EGC output SNR in equally correlated Nakagami- $m$ fading channels. The moments are expressed as the Appell hypergeometric functions and certain transformation formulas are used to ensure their convergence. Numerical results in Section 4 illustrate the effect of fading correlation on the distribution of the EGC output SNR. Section 5 concludes this paper.

\section{System And Channel Model}

Consider a diversity system with $L$ input branches. The received signal after the matched filter at the $k$-th branch can be written as

$$
y_{k}=g_{k} s+n_{k}, \quad k \in\{1, \ldots, L\},
$$


where $g_{k}$ is the random channel gain associated with the $k$-th branch, $s$ is the transmitted signal with energy $E_{s}$ and $n_{k}$ is the additive white Gaussian noise (AWGN) with power spectral density $\frac{N_{0}}{2}$ per dimension. We assume that the noise components are independent of the channel gains, i.e., $E\left(n_{j} G_{k}^{*}\right)=0$ for any $j, k \in\{1, \ldots, L\}$, where $x^{*}$ and $E(x)$ denote the complex conjugate and the average of $x$, and uncorrelated with each other, i.e., $E\left(n_{j} n_{k}^{*}\right)=N_{0} \delta_{j k}$, where $\delta_{j k}$ is the Kronecker delta function defined as $\delta_{j j}=1$ and $\delta_{j k}=0$ for $j \neq k$. Throughout this paper, all RVs are denoted by capital letters and the corresponding realizations are denoted by small letters.

In EGC, the received signals are co-phased and equally weighted, and then summed to form the resultant output. The instantaneous SNR at the EGC output can be written as

$$
\gamma_{e g c}=\frac{\left(r_{1}+r_{2}+\cdots+r_{L}\right)^{2} E_{s}}{L N_{0}}
$$

where $R_{k}=\left|G_{k}\right|$ is the amplitude of the channel gain $G_{k}$. We assume that the channel gains are equally correlated Nakagami- $m$ distributed with identical fading severity parameters $m$ 's at different diversity branches.

The Nakagami- $m$ distribution is a versatile statistical distribution which can accurately model a variety of fading environments. It has greater flexibility in matching some empirical data than the Rayleigh, Ricean or Lognormal distribution. It also includes the Rayleigh and the one-sided Gaussian distributions as special cases. Moreover, the Nakagami- $m$ distribution can closely approximate the Ricean and the Hoyt distributions [15]. When $m$ is an integer, the equally correlated Nakagami- $m$ envelopes can also be represented using a set of zero-mean complex Gaussian RVs

$$
G_{k j}=\left(\sqrt{1-\rho} X_{k j}+\sqrt{\rho} X_{0 j}\right)+i\left(\sqrt{1-\rho} Y_{k j}+\sqrt{\rho} Y_{0 j}\right)
$$

for $k \in\{1, \ldots, L\}$ and $j \in\{1, \ldots, m\}$, where $i=\sqrt{-1}$, $0 \leq \rho \leq 1,\left\{X_{k j}\right\}$ and $\left\{Y_{k j}\right\}, k \in\{0,1, \ldots, L\}$ and $j \in\{1, \ldots, m\}$, are two sets of independent zero-mean Gaussian RVs with identical variance $\frac{1}{2}$. That is, for any $j, k \in$ $\{0, \ldots, L\}, E\left(X_{k} Y_{j}\right)=0$ and $E\left(X_{k} X_{j}\right)=E\left(Y_{k} Y_{j}\right)=\frac{1}{2} \delta_{k j}$.

The fading correlation between any $G_{k j}$ and $G_{l n}$ is given by

$$
\rho_{g}=\frac{E\left(G_{k j} G_{l n}^{*}\right)}{\sqrt{E\left(\left|G_{k j}\right|^{2}\right) E\left(\left|G_{l n}\right|^{2}\right)}}= \begin{cases}\rho, & k \neq l \text { and } j=n, \\ 0, & j \neq n .\end{cases}
$$

Let $R_{k}^{2}$ denote the summation of the absolute square of $G_{k j}$ 's for $j=1, \ldots, m$,

$$
R_{k}^{2}=\sum_{j=1}^{m}\left|G_{k j}\right|^{2} .
$$

From (4), we find that for any fixed $k, G_{k j}$ is independent complex Gaussian distributed for any $j \in\{1, \ldots, m\}$. Thus $R_{k}^{2}$ is the sum of squares of $m$ independent Rayleigh envelopes with constant cross-correlation coefficient (power correlation) given by [15]

$$
\rho_{\eta}=\frac{E\left(R_{k}^{2} R_{l}^{2}\right)}{\sqrt{E\left(R_{k}^{4}\right) E\left(R_{l}^{4}\right)}}=\rho^{2}, \quad k \neq l .
$$

Therefore, $\left\{R_{k}\right\}$ is a set of equally correlated Nakagami-m fading envelopes with the mean-square value

$$
E\left(R_{k}^{2}\right)=m \text {. }
$$

\section{Output Moments}

Using the multinomial expansion, we obtain the moments of the EGC output SNR as

$$
\begin{aligned}
m_{n} & =E\left(\gamma_{\text {egc }}^{n}\right)=\left[\frac{E_{s}}{L N_{0}}\right]^{n} E\left[\left(R_{1}+R_{2}+\cdots+R_{L}\right)^{2 n}\right] \\
& =(2 n) !\left[\frac{\bar{\gamma}_{c}}{L E\left(R_{k}^{2}\right)}\right]^{n} \sum_{\substack{k_{1}, \cdots, k_{L}=0 \\
k_{1}+\cdots+k_{L}=2 n}}^{2 n} \frac{E\left(\prod_{j=1}^{L} R_{j}^{k_{j}}\right)}{\prod_{j=1}^{L} k_{j} !}
\end{aligned}
$$

where $\bar{\gamma}_{c}=\frac{E_{s}}{N_{0}} E\left(R_{k}^{2}\right)$ is the average branch SNR. When the channel gain envelopes $R_{k}$ 's are independent, (8) can be readily evaluated as

$$
m_{n}=(2 n) !\left[\frac{\bar{\gamma}_{c}}{L E\left(R_{k}^{2}\right)}\right]^{n} \sum_{\substack{k_{1}, \cdots, k_{L}=0 \\ k_{1}+\cdots+k_{L}=2 n}}^{2 n} \prod_{j=1}^{L}\left[\frac{E\left(R_{j}^{k_{j}}\right)}{k_{j} !}\right] .
$$

However, it is an extremely complicated task to compute (8) when the channel gains are correlated and the corresponding joint pdf is unknown.

Now the new channel gain representations (3) and (5) can be used to derive the moments of the EGC output SNR. Fix $X_{0 j}=x_{0 j}$ and $Y_{0 j}=y_{0 j}$ for $j \in\{1, \ldots, m\}$ and let $u=\sum_{j=1}^{m}\left(x_{0 j}^{2}+y_{0 j}^{2}\right)$, then $R_{k}^{2}$ is independent noncentral chi-square distributed with $2 m$ degrees of freedom and noncentrality parameter $\rho u$. The moments of $R_{k}$ can be found as [16, Eq. (2-1-146)]

$$
E\left(R_{i}^{k_{i}}\right)=(1-\rho)^{\frac{k_{i}}{2}} \frac{\Gamma\left(m+\frac{k_{i}}{2}\right)}{\Gamma(m)} \Phi\left(-\frac{k_{i}}{2}, m ;-\frac{\rho u}{1-\rho}\right)
$$

where $\Gamma(x)$ is the gamma function and $\Phi(a, c ; z)$ is the confluent hypergeometric function defined as [17, Eq. (9.210.1)].

Substituting (7) and (10) into (9), we obtain the output moments of EGC conditioned on $u$ as

$$
\begin{aligned}
& m_{n}(u)=(2 n) !\left[\frac{\bar{\gamma}_{c}(1-\rho)}{m L}\right]^{n} \\
& \times \sum_{\substack{k_{1}, \cdots, k_{L}=0 \\
k_{1}+\cdots+k_{L}=2 n}}^{2 n}\left[\prod_{j=1}^{L} B\left(k_{j}\right) \Phi\left(-\frac{k_{j}}{2}, m ;-\frac{\rho u}{1-\rho}\right)\right]
\end{aligned}
$$

where

$$
B\left(k_{j}\right)=\frac{\Gamma\left(m+\frac{k_{j}}{2}\right)}{(m-1) ! k_{j} !} .
$$

Also notice that $U$ is chi-square distributed with $2 m$ degrees 
of freedom and its pdf is given by [16]

$$
p_{U}(u)=\frac{1}{\Gamma(m)} u^{m-1} e^{-u}, \quad u \geq 0 .
$$

Averaging the conditional output moments (11) over the distribution of $U$ (13), we obtain the moments of the EGC output SNR in equally correlated Nakagami- $m$ fading channels as

$$
\begin{aligned}
& m_{n}=(2 n) !\left[\frac{\bar{\gamma}_{c}(1-\rho)}{m L}\right]^{n} \\
& \times \sum_{\substack{k_{1}, \cdots, k_{L}=0 \\
k_{1}+\cdots+k_{L}=2 n}}^{2 n}\left[K\left(k_{1}, \ldots, k_{L}\right) \prod_{i=1}^{L} B\left(k_{j}\right)\right]
\end{aligned}
$$

where $K\left(k_{1}, \ldots, k_{L}\right)$ is a single-fold integral which can be derived in terms of the $L$-th order Appell hypergeometric function with the aid of [18, Eq. (C.1)]:

$$
\begin{aligned}
K & \left(k_{1}, \ldots, k_{L}\right) \\
\quad & =\int_{0}^{\infty} \frac{u^{m-1} e^{-u}}{\Gamma(m)} \prod_{j=1}^{L} \Phi\left(-\frac{k_{j}}{2}, m ;-\frac{\rho u}{1-\rho}\right) d t \\
& =F_{A}\left(m ;-\frac{k_{1}}{2}, \cdots,-\frac{k_{L}}{2} ; m, \cdots, m ; x_{1}, \ldots, x_{L}\right)
\end{aligned}
$$

where $x_{k}=-\frac{\rho}{1-\rho}$ for $k \in\{1, \ldots, L\}$ and $F_{A}\left(\alpha ; \beta_{1}, \ldots, \beta_{n} ; \gamma_{1}, \ldots, \gamma_{n} ; x_{1}, \ldots, x_{n}\right)$ is the $n$-th order Appell hypergeometric function defined as [17, (9.180.2)]

$$
\begin{aligned}
& F_{A}\left(\alpha ; \beta_{1}, \ldots, \beta_{n} ; \gamma_{1}, \ldots, \gamma_{n} ; x_{1}, \ldots, x_{n}\right)= \\
& \sum_{p_{1}=0}^{\infty} \cdots \sum_{p_{n}=0}^{\infty} \frac{(\alpha)_{p_{1}+\cdots+p_{n}}\left(\beta_{1}\right)_{p_{1}} \cdots\left(\beta_{n}\right)_{p_{n}}}{\left(\gamma_{1}\right)_{p_{1}} \cdots\left(\gamma_{n}\right)_{p_{n}} p_{1} ! \cdots p_{n} !} x_{1}^{p_{1}} \cdots x_{n}^{p_{n}}
\end{aligned}
$$

whose convergence region is defined by $\left|x_{1}\right|+\ldots+\left|x_{L}\right|<1$ [19]. If all $\beta_{j}$ 's are either zeros or negative integers, then the Appell hypergeometric function (16) reduces to a finite series which is given by

$$
\begin{aligned}
& F_{A}\left(\alpha ; \beta_{1}, \cdots, \beta_{n} ; \gamma_{1}, \cdots, \gamma_{n} ; x_{1}, \cdots, x_{n}\right)= \\
& \sum_{p_{1}=0}^{-\beta_{1}} \cdots \sum_{p_{n}=0}^{-\beta_{n}} \frac{(\alpha)_{p_{1}+\cdots+p_{n}}\left(\beta_{1}\right)_{p_{1}} \cdots\left(\beta_{n}\right)_{p_{n}}}{\left(\gamma_{1}\right)_{p_{1}} \cdots\left(\gamma_{n}\right)_{p_{n}} p_{1} ! \cdots p_{n} !} x_{1}^{p_{1}} \cdots x_{n}^{p_{n}} .
\end{aligned}
$$

Notice that (15) only converges for $-\frac{1}{L-1}<\rho<\frac{1}{L+1}$. Hence a transformation formula for the higher transcendental function $F_{A}$ [19] must be used to obtain a converge expression for (15) as

$$
\begin{aligned}
& K\left(k_{1}, \ldots, k_{L}\right)=\frac{1}{(m-1) !}\left[\frac{1-\rho}{1+(L-1) \rho}\right]^{m} \\
& \quad \times F_{A}\left(m ; \beta_{1}, \cdots, \beta_{L} ; m, \cdots, m ; z_{1}, \ldots, z_{L}\right) .
\end{aligned}
$$

where $\beta_{j}=m+\frac{k_{j}}{2}$ and $z_{j}=\frac{\rho}{1+(L-1) \rho}$ for $j \in\{1, \ldots, L\}$. Since $0 \leq \rho<1,\left|z_{1}\right|+\ldots+\left|z_{L}\right|=\frac{L \rho}{L \rho+(1-\rho)}<1$, i.e., the Appell hypergeometric function $F_{A}$ in (18) converges for any $0 \leq \rho<1$. Combining (18) with (12) and (14), we can readily compute the moments of the $L$-branch EGC output SNR in equally correlated Nakagami- $m$ fading channels.
When $m=1$, we obtain the moments of the EGC output in equally correlated Rayleigh fading channels as

$$
\begin{aligned}
& m_{n}=(2 n) !\left[\frac{\bar{\gamma}_{c}(1-\rho)}{L}\right]^{n} \\
& \times \sum_{\substack{k_{1}, \cdots, k_{L}=0 \\
k_{1}+\cdots+k_{L}=2 n}}^{2 n}\left[I\left(k_{1}, \ldots, k_{L}\right) \prod_{j=1}^{L} A\left(k_{j}\right)\right]
\end{aligned}
$$

where

$$
A\left(k_{j}\right)=\frac{\Gamma\left(1+\frac{k_{j}}{2}\right)}{k_{j} !}
$$

and

$$
\begin{aligned}
& I\left(k_{1}, \ldots, k_{L}\right)=\frac{1-\rho}{1+(L-1) \rho} \\
& \quad \times F_{A}\left(1 ; \beta_{1}, \cdots, \beta_{L} ; 1, \cdots, 1 ; z_{1}, \ldots, z_{L}\right)
\end{aligned}
$$

where $\beta_{j}=1+\frac{k_{j}}{2}$ and $z_{j}=\frac{\rho}{1+(L-1) \rho}$ for $j \in\{1, \ldots, L\}$.

Next we present some special cases of (14) and (19).

a) Average Output SNR: Using (17) and the relation between the second kind Appell hypergeometric function and the Gauss hypergeometric function [18, Eq. (C.4)], we can readily simplify the average output SNR of EGC in equally correlated Rayleigh and Nakagami- $m$ fading channels respectively as

$$
\bar{\gamma}_{e g c}=m_{1}=\bar{\gamma}_{c}\left[1+\frac{(L-1) \pi}{4}{ }_{2} F_{1}\left(-\frac{1}{2},-\frac{1}{2} ; 1 ; \rho^{2}\right)\right]
$$

and

$$
\bar{\gamma}_{e g c}=\bar{\gamma}_{c}\left[1+\frac{(L-1) \Gamma^{2}\left(m+\frac{1}{2}\right)}{m[(m-1) !]^{2}} F_{1}\left(-\frac{1}{2},-\frac{1}{2} ; m ; \rho^{2}\right)\right]
$$

where ${ }_{2} F_{1}(a, b ; c ; z)$ is the Gauss hypergeometric function defined as [17, Eq. (9-100)].

When $\rho=0$, (22) reduces to

$$
\bar{\gamma}_{e g c}=\bar{\gamma}_{c}\left[1+\frac{(L-1) \pi}{4}\right] \text {, }
$$

which is the well-known result for independent Rayleigh fading channels [20, Eq. (5.2-20)].

b) Independent Fading: In the independent case $(\rho=0)$, (19) and (14) reduce to

$$
m_{n}=\left(\frac{\bar{\gamma}_{c}}{L}\right)^{n}(2 n) ! \sum_{\substack{k_{1}, \cdots, k_{L}=0 \\ k_{1}+\cdots+k_{L}=2 n}}^{2 n}\left[\prod_{j=1}^{L} A\left(k_{j}\right)\right] .
$$

and

$$
m_{n}=\left(\frac{\bar{\gamma}_{c}}{m L}\right)^{n}(2 n) ! \sum_{\substack{k_{1}, \cdots, k_{L}=0 \\ k_{1}+\cdots+k_{L}=2 n}}^{2 n}\left[\prod_{j=1}^{L} B\left(k_{j}\right)\right],
$$

for Rayleigh and Nakagami- $m$ fading channels respectively.

c) Dual-branch EGC in Equally Correlated Rayleigh Fading: The moments of the dual-branch EGC output SNR in equally correlated Rayleigh fading channels can be written 
in terms of the Gauss hypergeometric function as

$$
\begin{aligned}
m_{n} & =\left(\frac{\bar{\gamma}_{c}}{2}\right)(2 n) ! \sum_{k=0}^{2 n} \frac{\Gamma\left(1+\frac{k}{2}\right) \Gamma\left(1+n-\frac{k}{2}\right)}{k !(2 n-k) !} \\
& \times{ }_{2} F_{1}\left(-\frac{k}{2},-\frac{2 n-k}{2} ; 1 ; \rho^{2}\right) .
\end{aligned}
$$

As comparison, we present the moments of the MRC output SNR in equally correlated Rayleigh fading channels here. The pdf of the MRC output SNR in such channels is given by [13, Eq. (12)]. Hence, the output moments are obtained as

$$
\begin{aligned}
E\left(\gamma_{m r c}^{n}\right) & =\bar{\gamma}_{c}^{n}\left[\frac{(1-\rho)^{1+n} \Gamma(L+n)}{(1-\rho+L \rho) \Gamma(L)}\right. \\
& \left.\times{ }_{2} F_{1}\left(1, L+n ; L ; \frac{L \rho}{1-\rho+L \rho}\right)\right] .
\end{aligned}
$$

The moments of the EGC output SNR can be further used to evaluate the average error rate and the outage performance of multi-branch EGC in equally correlated Nakagami- $m$ fading channels with the aid of the Edgeworth series and Gaussian quadrature techniques. We can also derive some purely moment-based measures, such as the skewness, the kurtosis and the Karl Pearson's coefficient of variation. For brevity, we do not develop such results here. Detailed discussion can be found in [21].

\section{NumERICAL RESUlts}

We now present numerical results to show the correlation effect on the distribution of the EGC output SNR. Semianalytical simulations are provided as an independent check of our analytical results.

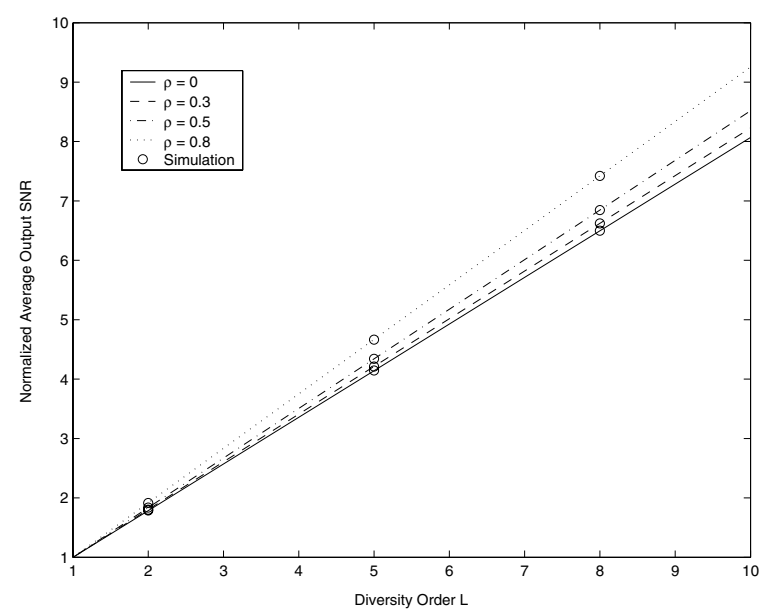

Fig. 1. The average output SNR of EGC in equally correlated Rayleigh fading channels.

Fig. 1 plots the normalized average output SNR $\hat{\gamma}_{e g c}=$ $\gamma_{e g c} / \bar{\gamma}_{c}$ of EGC versus the diversity order $L$ in equally correlated Rayleigh fading channels. As the diversity order $L$ increases, the average output SNR of EGC increases. Fig. 2 plots the normalized average output SNR of 4-branch EGC

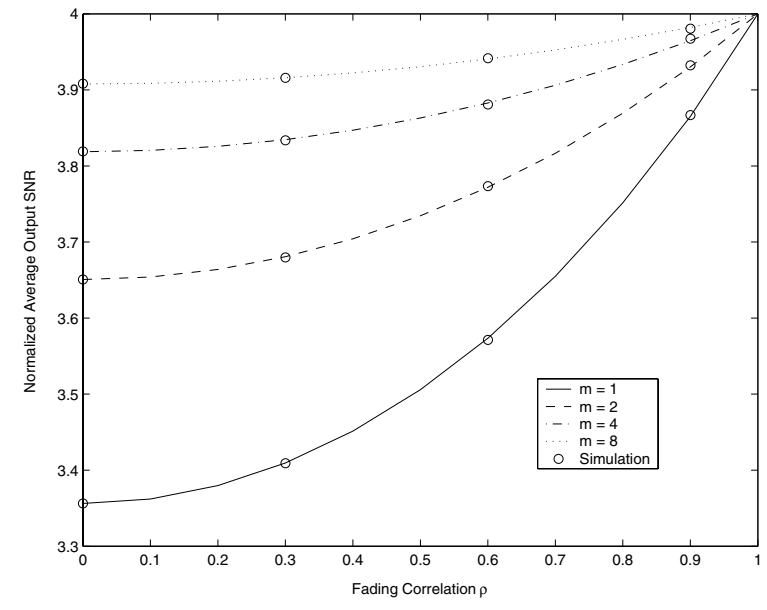

Fig. 2. The average output SNR of 4-branch EGC in equally correlated Nakagami- $m$ fading channels.

versus the fading correlation $\rho$ in equally correlated Nakagami$m$ fading channels. As the fading figure $m$ increases, i.e., the channel fading condition improves, the average output SNR increases and the fading correlation has less effect on the EGC output. In both figures, we find that the average output SNR also increases as the fading correlation $\rho$ increases. This has never happened in the MRC and SC performance [3]. This observation departs from our expectations. However, it can be explained by (8). Since $E\left(R_{j} R_{k}\right)>E\left(R_{j}\right) E\left(R_{k}\right),(j \neq k)$, for $\rho>0$, the average output SNR of EGC in correlated fading channels is higher than that in independent fading channels [3]. In fact. as $\rho$ approaches 1 , the average output SNR of EGC approaches that of MRC as

$$
\bar{\gamma}_{e g c}=\bar{\gamma}_{m r c}=L \bar{\gamma}_{c}
$$

It should be noted that common EGC performance measures, such as BER, cannot be solely characterized by the average output SNR; they also depend on the higher-order moments. The average output SNR by itself is not a comprehensive metric for the EGC performance in correlated fading channels. Caution must be exercised when using the average output SNR as a performance measure. Higher-order moment based performance measures of the combiner output SNR are required.

Charash [22] introduced the square value of the Karl Pearson's coefficient as a unified measure of the amount of fading (AF)

$$
\mathrm{AF}=\frac{m_{2}}{m_{1}^{2}}-1
$$

Using (19) and (14), we may obtain the AF of EGC in equally correlated Rayleigh and Nakagami- $m$ fading channels. For example, the AF of the dual-branch EGC output in equally correlated Rayleigh fading channels is obtained as

$$
\mathrm{AF}=\frac{\frac{5}{2}+\frac{3}{2} \rho^{2}+\frac{3 \pi}{4}{ }_{2} F_{1}\left(-\frac{3}{2},-\frac{1}{2} ; 1 ; \rho^{2}\right)}{\left[1+\frac{\pi}{4}{ }_{2} F_{1}\left(-\frac{1}{2},-\frac{1}{2} ; 1 ; \rho^{2}\right)\right]^{2}}-1 .
$$

Fig. 3 compares the AF of EGC with that of MRC in equally correlated Rayleigh fading channels. As expected, the 


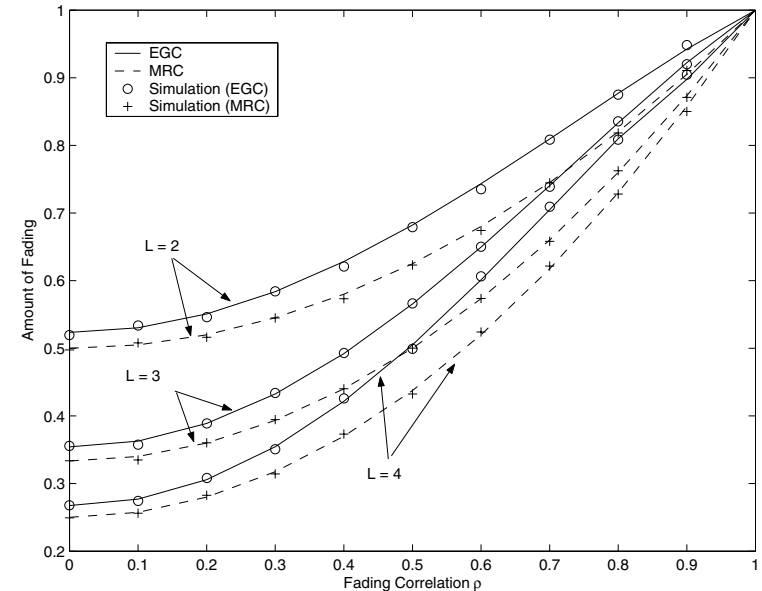

Fig. 3. The amount of fading of EGC in equally correlated Rayleigh fading channels.

$\mathrm{AF}$ increases as the fading correlation $\rho$ increases and the diversity order $L$ decreases. The AF of EGC is larger than that of MRC. Compared to the average output SNR, AF is a more reliable performance measure because it takes into consideration the second moment of the output SNR.

\section{CONCLUSION}

In this paper, we developed a new representation for the equally correlated Nakagami- $m$ channel gains. Using the new channel gain representation, we have derived the moments of the EGC output SNR in equally correlated Nakagami- $m$ fading channels. Moment-based analysis techniques can serve as an alternative to the more common pdf and mgf approaches. We derived some purely moment-based measures, such as the average output SNR and the amount of fading. Numerical and simulation results show that the fading correlation has a negative effect on the EGC performance. The average output SNR is not a complete performance measure. Higher-order moments may be required to study the EGC performance. Our moments expressions can also be used to evaluate the average error rate and the outage probability of EGC. These results can be found in our journal paper [21].

\section{REFERENCES}

[1] G. Taricco, E. M. Biglieri and G. Caire, "Impact of channel-state information on coded transmission over fading channels with diversity reception," IEEE Trans. Commun., vol. 47, pp. 1284-1287, Sept. 1999.

[2] N. C. Beaulieu, "An infinite series for the computation of the complementary probability distribution function of a sum of independent random variables and its application to the sum of Rayleigh random variables," IEEE Trans. Commun., vol. 38, pp. 1463-1474, Sept. 1990.

[3] D. G. Brennan, "Linear diversity combining techniques," Proc. IRE, vol. 47, pp. 1075-1102, June 1959.

[4] A. Annamalai, C. Tellambura, and V. K. Bhargava, "Exact evaluation of maximal-ratio and equal-gain diversity receivers for M-ary QAM on Nakagami fading channels," IEEE Trans. Commun., vol. 47, pp. 13351344, Sept. 1999.

[5] Q. T. Zhang, "Probability of error for equal-gain combiners over Rayleigh channels: some closed-form solutions," IEEE Trans. Commun., vol. 45, pp. 270-273, Mar. 1997.
[6] R. Mallik, M. Win, and J. Winters, "Performance of predetection dual diversity in correlated Rayleigh fading: EGC and SD," IEEE GLOBECOM, pp. 932-936, 2000.

[7] C. Tellambura and A. Annamalai, Wiley Encyclopedia of Telecommunications. Wiley, Jan 2003, ch. Wireless Communications Systems Design.

[8] A. Annamalai, V. Ramanathan and C. Tellambura, "Analysis of equalgain diversity receiver in correlated fading channels," in Proc. IEEE VTC Spring 2002, vol. 4, Birmingham, Al, May 2002, pp. 2038 -2041.

[9] C. D. Iskander and P. T. Mathiopoulos, "Performance of M-QAM with coherent equal-gain combining in correlated Nakagami- $m$ fading," Electronics letters, vol. 39, no. 1, pp. 141-142, Jan. 2003.

[10] G. K. Karagiannidis, D. A. Zogas and S. A. Kotsopoulos, "Statistical properties of the EGC output SNR over correlated Nakagami- $m$ fading channels," To appear in IEEE Trans. Wirel. Commun.

[11] Y. Chen and C. Tellambura, "Performance analysis of $l$-branch equal gain combiners in equally-correlated Rayleigh fading channels," IEEE Commun. Lett., vol. 8, pp. 150-152, Mar. 2004.

[12] — , "Performance of $L$-branch diversity combiners over equallycorrelated Rician fading channels," To appear in GLOBECOM 2004.

[13] V. A. Aalo, "Performance of maximal-ratio diversity systems in a correlated Nakagami-fading environment," IEEE Trans. Commun., vol. 43 , no. 8, pp. 2360-2369, Aug. 1995.

[14] M. Z. Win, R. K. Mallik and G. Chrisikos, "Higher order statistics of antenna subset diversity," IEEE Trans. Wirel. Commun., vol. 2, pp. 871875, Sept 2003

[15] M. Nakagami, "The m-distribution, a general formula of intensity distribution of rapid fading," in Statistical Methods in Radio Wave Propagation, W. G. Hoffman, Ed. Oxford, England: Pergamon, 1960.

[16] J. G. Proakis, Digital Communications, 3rd ed. New York: McGrawHill, 1995.

[17] I. S. Gradshteyn and I. M. Ryzhik, Table of Integrals, Series, and Products, 5th ed. Academic Press, Inc., 1994.

[18] A. Annamalai, C. Tellambura and V.K. Bhargava, "Equal-gain diversity receiver performance in wireless channels," IEEE Trans. Commun., vol. 48, pp. 1732-1745, Oct. 2000

[19] P. Appell and J. Kampé de Fériet, Fonctions Hypergéométriques et Hypersphériques; Polynomes d'Hermite. Paris: Gauthier-Villars, 1926.

[20] W. C. Jakes, Microwave Mobile Communications. New York: IEEE Press, 1994.

[21] Y. Chen and C. Tellambura, "Moments of the equal gain combiner output in equally correlated fading channels," Submitted to IEEE Trans. Veh. Technol.

[22] U. Charash, "Reception through Nakagami multipath channels with random delays," IEEE Trans. Commun., vol. 27, no. 4, pp. 657-670, Apr. 1979. 